Document downloaded from:

http://hdl.handle.net/10251/164224

This paper must be cited as:

Soldevila Serrano, S.; Bosca Mayans, F. (2020). Assessing physical properties of amphoteric fluoroquinolones using phosphorescence spectroscopy. Spectrochimica Acta Part A Molecular and Biomolecular Spectroscopy. 227:1-7. https://doi.org/10.1016/j.saa.2019.117569

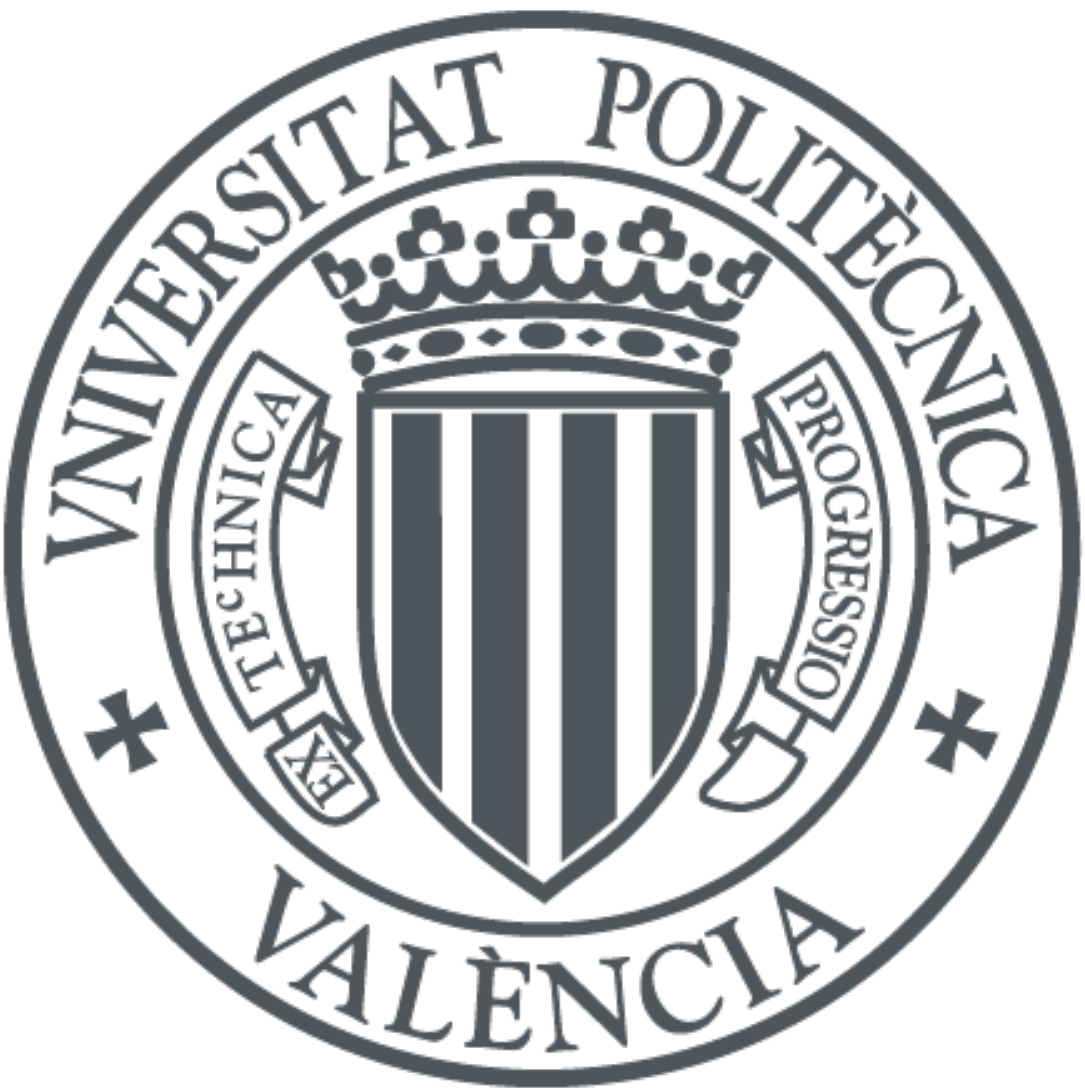

The final publication is available at

https://doi.org/10.1016/j.saa.2019.117569

Copyright Elsevier

Additional Information 


\title{
Assessing Physical Properties of Amphoteric Fluoroquinolones using phosphorescence spectroscopy
}

\author{
Sonia Soldevila, Francisco Bosca*
}

Instituto de Tecnología Química UPV-CSIC, Universitat Politècnica de València, Avda de los Naranjos, s/n, 46022 Valencia, Spain. E-mail: fbosca@itq.upv.es

ABSTRACT: The self-association of fluoroquinolones (FQ) in water would play a relevant role in their translocations across lipid membranes. Triplet excited states of these drugs have been shown as reporters of FQ self-association using laser flash photolysis technique. A study using low-temperature phosphorescence technique was performed with quinolone derivatives such as enoxacin (ENX), norfloxacin (NFX), pefloxacin (PFX), ciprofloxacin (CPX, ofloxacin (OFX), nalidixic acid (NLA), pipemidic acid (PPA) and piromidic acid (PRA) to explore emission changes associated with self-associations and to shed some light on the triplet excited state energy $\left(\mathrm{E}_{\mathrm{T}}\right)$ discrepancies described in the literature for most of these drugs. The emissions obtained at $77 \mathrm{~K}$ in buffered aqueous medium revealed that the amphoteric nature of the quinolones CPX, NFX, PFX, ENX, OFX and PPA must generate their self-associations because a redshift of their phosphorescence maxima is produced by FQ concentrations increases. Hence, this effect was not observed for NLA and PRA or when all quinolones were analysed using ethanol or ethylene glycol aqueous mixtures as glassed solvents. Interestingly, the presence of these organic mixtures produced a blue-shift in the phosphorescence emission maximum of each FQ. Additionally, laser flash photolysis experiments with PRA and the amphoteric quinolone PPA, compounds with the same skeleton but different peripheral substituent, confirm the expected correlations between the amphoteric nature of compounds and their self-associations in aqueous media because the excimer generation was only detected for PPA. Now, the discrepancies described in the literature for the $\mathrm{E}_{\mathrm{T}}$ of FQs can be understood considering that changes of medium polarity or proticity as well as the temperature can considerably modify their $\mathrm{E}_{\mathrm{T}}$ values. Thereby, low-temperature phosphorescence technique, is an effective way to 
detect molecular self-associations and surrounding changes in quinolones that opens the possibility to evaluate these effects in other drug families.

\section{Keywords.}

fluoroquinolones, laser flash photolysis, excimers, triplet energy, phosphorescence, self-associations

\section{Highlights.}

- Low-temperature phosphorescence technique to detected molecular self-associations

- Substantial changes of quinolones phosphorescence by polarity/proticity of medium

- Amphoteric quinolones form self-associations in buffered water.

- Generation of triplet excimers from self-associations of quinolone derivatives 


\section{Graphical abstract.}
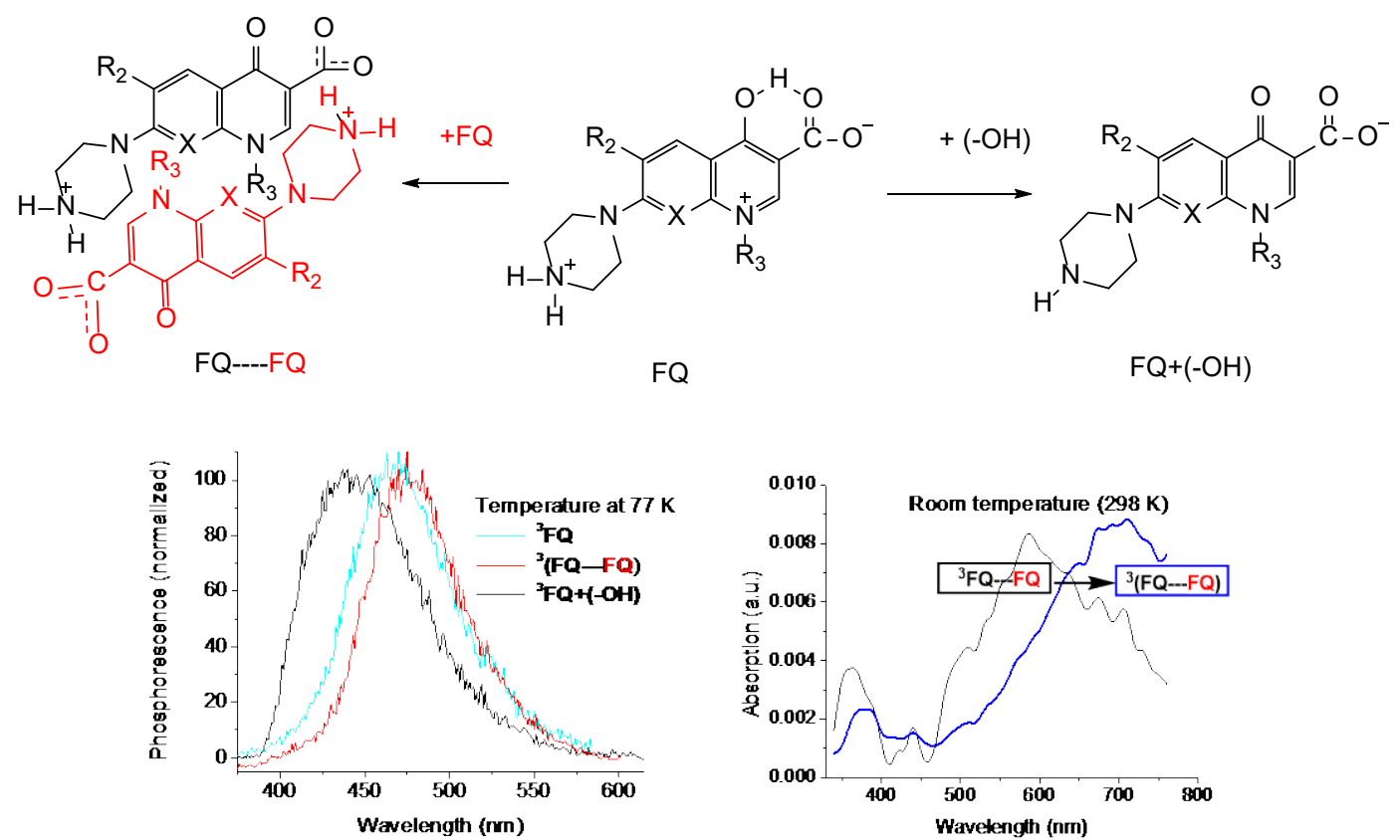


\section{Introduction}

Fluoroquinolones (FQs) are antibacterial agents developed by modifications of nalidixic acid (NLA, see

Chart 1). The potent activity of NLA against Gram-negative bacteria has been greatly improved by combining a 7-piperazinyl ring and a 6-fluorine atom on the quinolone nucleus as in the case of ciprofloxacin (CPX), norfloxacin (NFX) and pefloxacin (PFX) or on the 1,8-naphthyridine nucleus (such as enoxacin, ENX), or producing fluorinated tricyclic derivatives (ofloxacin, OFX). These drugs have generated a great interest during the last forty years mainly due to their broad antibacterial spectrum with less resistance rapid effect and good oral absorption properties. [1, 2] The antibacterial activity of FQs appears to result from combination efficient cellular membrane penetration and DNA gyrase inhibiting activity.[3] Despite the predominant zwitterionic character of FQs at neutral $\mathrm{pH}$, they are able to achieve high concentrations in cells.[4-5] The results of different studies of membrane permeability have proven that the main pathway to permeate the FQs through the membrane is the passive diffusion. This view have been also supported by various membrane vesicle and liposome experiments. [6-7] It has been suggested that the FQ zwitterionic species is responsible for the diffusion through the cellular membrane. However, although the mechanism is still under debate, a theoretical study has shown a novel molecular entry pathway that involves dissolution of concerted stacks of the zwitterionic form of CPX for the translocation of this amphoteric drug across lipid membranes.[3] The occurrence of FQ selfassociations in aqueous media has also been proposed to explain the generation of triplet eximers in water from the reaction of FQ triplet excited states $\left({ }^{3} \mathrm{FQs}\right)$ with their ground states at very low concentrations (below $\left.10^{-4} \mathrm{M}\right)$.[8] Thereby, fluoroquinolone triplet excited states ( ${ }^{3} \mathrm{FQs}$ ) have been shown as reporters of FQ self-association using laser flash photolysis technique. In this context, drugbiomolecules interactions including FQs-DNA associations have been also studied analysing the reactivity of the triplet excited state with the surrounding [9-11]. Interestingly, low-temperature phosphorescence technique has been used to determine properties of triplet excited states of drugs such as their energies and also has been widely used to detect stacking interactions of drugs with DNA.[11-13] Nev- 
ertheless, there are relevant discrepancies in the literature about the energies of ${ }^{3} \mathrm{FQs}$ using this technique as well as by theoretical methods or analysing energy transfer reactions in laser flash photolysis studies.[11,14-21]

With this background, low-temperature phosphorescence measurements were performed with FQs (CPX, NFX, PFX, ENX, OFX) and other structurally simplest quinolone derivatives such as NLA, pipemidic and piromicic acids (PPA, PRA respectively, see structures in Chart 1) to explore emission changes due to their expected self-associations. Moreover, it was also tried to shed some light on the relevant discrepancies about the energies of ${ }^{3} \mathrm{FQs},[11,14-21]$ which are crucial for the understanding of the photomutagenic properties associated with these drugs.[22-23] For those purpose, the quinolone derivatives were analysed using low-temperature phosphorescence at several concentrations in different media. Additionally, laser flash photolysis experiments were also performed with the very close related quinolone derivatives PPA and PRA for proving the correlation between the amphoteric nature of a quinolone derivative, its self-association in aqueous media and the excimer detection.
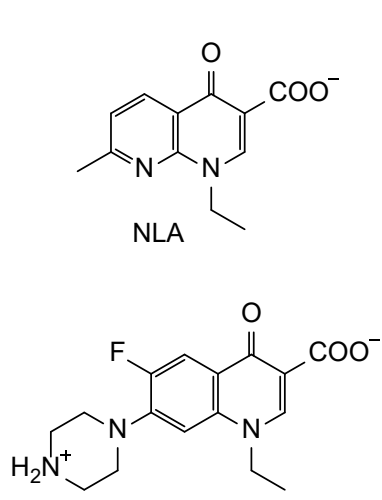

NFX

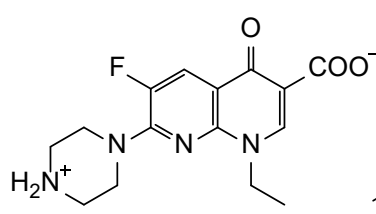

ENX
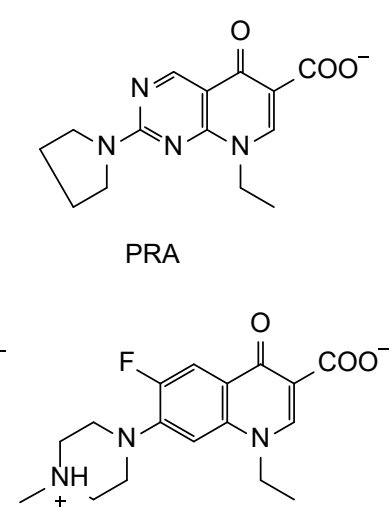

PFX

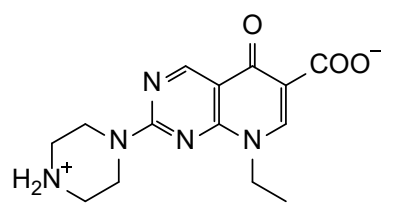

PPA

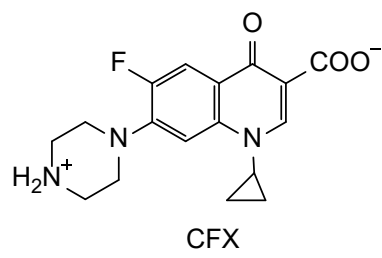

Chart 1. Structure of quinolone derivatives under study. 


\section{Experimental:}

\subsection{Reagents.}

Ciprofloxacin (CPX), norfloxacin (NFX), pefloxacin (PFX), enoxacin (ENX), ofloxacin (OFX), nalidixic acid (NLA), pipemidic acid (PPA) and piromicic acids (PRA) were commercial products obtained from Sigma-Aldrich Chemical Company. Sodium phosphate buffer (PB) were prepared from reagentgrade products using milli-Q water; the $\mathrm{pH}$ of the solutions was measured through a glass electrode and adjusted with $\mathrm{NaOH}$ to the different pHs. Other chemicals were reagent grade and were used as received.

The aqueous samples of fluoroquinolones (FQs) as well as NLA, PPA and PRA were prepared with phosphate buffer (PB) using a mother solution of $300 \mathrm{mM} \mathrm{PB}$ adjusted at $\mathrm{pH} 7.4$ with a Crison $\mathrm{pH}-$ meter.

\subsection{Instrumentation.}

UV-Vis absorption spectra were registered on a Cary 50 spectrophotometer (Varian, Palo Alto, CA, USA) using a quartz cuvette of $3 \mathrm{~mL}$ and a $1 \mathrm{~cm}$ optical path.

The low-temperature phosphorescence emission spectra were registered with a Photon Technology International (PTI, TimeMaster TM/2003, Birmingham, NJ, USA) equipped with a pulsed Xenon lamp. The laser flash photolysis (LFP) experiments were run with a pulsed Nd:YAG (L52137 V LOTIS TII, Minsk, Belarus) laser system instrument setting $355 \mathrm{~nm}$ as the excitation wavelength. The pulse duration was of ca. $10 \mathrm{~ns}$, and the energy was adjusted at $10 \mathrm{~mJ} /$ pulse. The apparatus consisted of the pulsed laser, a $150 \mathrm{~W}$ Xe lamp, a 77250 Oriel monochromator, and a photomultiplier. The output signal from a Tektronix (Les Ulis, France) oscilloscope was transferred to a personal computer. The transient spectra were recorded at room temperature, employing quartz cells of $1 \mathrm{~cm}$ optical path length. 


\subsection{Procedures}

\subsubsection{Phosphorescence spectra.}

All compounds were studied at $5 \times 10^{-6}$ and $10^{-4} \mathrm{M}$ concentrations using three different solvents; a) 2 $\mathrm{mM}$ phosphate buffered $(\mathrm{PB})$ aqueous solution adjusted at $\mathrm{pH}$ ca. 7.4, b) a mixture of solvent a)/ethanol $(1 / 2), c)$ a mixture of solvent a)/ethylene glycol $(1 / 2)$. In the case of CFX, the study was extended to measurements at different pHs. Thus, for this purpose, initially solvent a) was adjusted to pHs 12 and 4 adding $\mathrm{NaOH}(1 \mathrm{M})$ and $\mathrm{HCl}(1 \mathrm{M})$ to the $2 \mathrm{mM}$ phosphate buffered $(\mathrm{PB})$ aqueous solution and these new solvents were used to prepare the acidic and basic organic polar mixtures. Finally, the solutions were transferred to quartz tubes of $5 \mathrm{~mm}$ diameter to perform the measurements at $77 \mathrm{~K}$. The excitation $(330 \mathrm{~nm})$ and the emission bandwidths $(375-650 \mathrm{~nm})$ were both set to $5 \mathrm{~nm}$.

\subsubsection{Laser flash photolysis.}

The studies of PPA and PRA were performed at $10^{-4} \mathrm{M}$ concentrations in buffered aqueous solutions under aerobic (in air) and anaerobic conditions (by bubbling $\mathrm{N}_{2} \mathrm{O}$ ).

Determination of the rate constants for quenching $(\mathrm{kq})$ of triplet excited state PPA and PRA by biphenyl carboxylic acid (BPC) through an energy transfer reaction between ${ }^{3} \mathrm{PPA}$ or ${ }^{3} \mathrm{PRA}$ and $\mathrm{BPC}$ ground state (see equation 1) were carried out using buffered aqueous solutions of PPA and PRA with increasing amounts of the quenchers BPC $(0.1-10 \mathrm{mM})$ ensuring that no changes in the $\mathrm{pH}$ were induced. Under these conditions more than $99 \%$ of the light was absorbed by the fluoroquinolone.

$$
{ }^{3} \mathrm{PPA}+\mathrm{BPC} \longrightarrow \mathrm{PPA}+{ }^{3} \mathrm{BPC}
$$




\section{Results}

3.1. Effect of concentration and polariry/proticity of medium in the phosphorescence of quinolone derivatives.

Low temperature phosphorescence spectra of ciprofloxacin (CPX), norfloxacin (NFX), pefloxacin (PFX), enoxacin (ENX), ofloxacin (OFX), nalidixic acid (NLA), pipemidic acid (PPA) and piromicic acids (PRA) were performed at $5 \times 10^{-6}$ and $5 \times 10^{-4} \mathrm{M}$ concentrations in $2 \mathrm{mM} \mathrm{PB}$ aqueous solutions adjusted at $\mathrm{pH}$ ca. 7.4. Interestingly, all of them excluding NLA and PRA showed a redshift of their emission maxima.
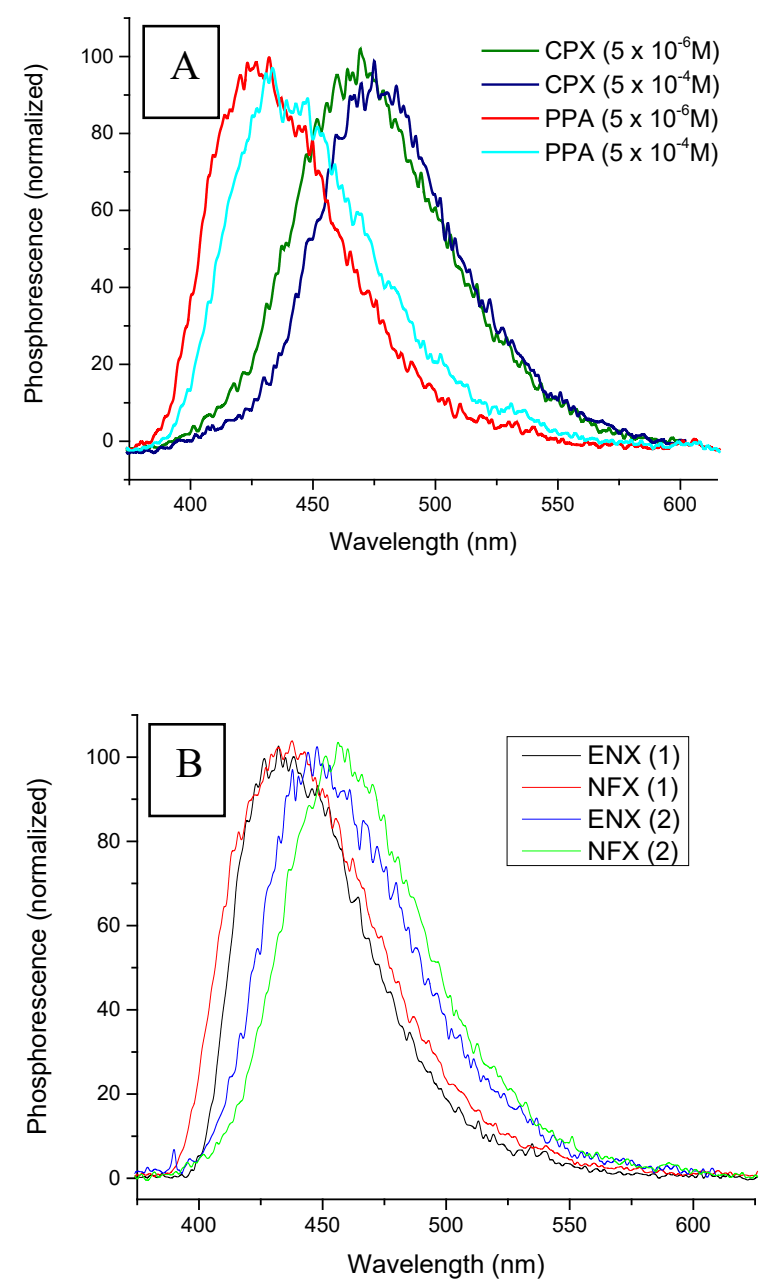
Figure 1. A) Normalized phosphorescence emissions of CPX and PPA at $5 \times 10^{-6} \mathrm{M}$ and $5 \times 10^{-4} \mathrm{M}$ in buffered water at pH 7.4 (2mM PB) at 77 K. B) Normalized phosphorescence emissions of NFX and ENX at $5 \times 10^{-6} \mathrm{M}$ in buffered water at $\mathrm{pH} 7.4$ (1) and in $2 \mathrm{mM} \mathrm{PB} / \mathrm{ethanol}$ at $77 \mathrm{~K}$.

Table 1. Low-temperature phosphorescence data of quinolone derivatives in different media and at different concentrations.

\begin{tabular}{|l|l|l|l|l|l|l|l|l|}
\hline \multicolumn{2}{|c|}{$\lambda_{\max }{ }^{\mathrm{a}}, \mathrm{nm}\left(\mathrm{E}_{\mathrm{T}}, \mathrm{kJ} / \mathrm{mol}\right)^{\mathrm{b}}$} \\
\hline & NFX & CPX & PFX & ENX & OFX & NLA & PRA & PPA \\
\hline $\begin{array}{l}2 \mathrm{mM} \mathrm{PB} \\
\left(5 \times 10^{-6} \mathrm{M}\right)\end{array}$ & $\begin{array}{l}463 \\
(288)\end{array}$ & $\begin{array}{l}465 \\
(285)\end{array}$ & $\begin{array}{l}460 \\
(289)\end{array}$ & $\begin{array}{l}442 \\
(293)\end{array}$ & $\begin{array}{l}507 \\
(270)\end{array}$ & $\begin{array}{l}410 \\
(311)\end{array}$ & $\begin{array}{l}435 \\
(293)\end{array}$ & $\begin{array}{l}421 \\
(303)\end{array}$ \\
\hline $\begin{array}{l}2 \mathrm{mM} \mathrm{PB} \\
\left(5 \mathrm{x} 10^{-4} \mathrm{M}\right)\end{array}$ & $\begin{array}{l}473 \\
(283)\end{array}$ & $\begin{array}{l}475 \\
(281)\end{array}$ & $\begin{array}{l}470 \\
(284)\end{array}$ & $\begin{array}{l}458 \\
(287)\end{array}$ & $\begin{array}{l}510 \\
(269)\end{array}$ & $\begin{array}{l}410 \\
(311)\end{array}$ & $\begin{array}{l}435 \\
(293)\end{array}$ & $\begin{array}{l}430 \\
(298)\end{array}$ \\
\hline $\begin{array}{l}2 \mathrm{mM} \mathrm{PB} / \\
\text { Etanol (1/2) }\end{array}$ & $\begin{array}{l}430 \\
(302)\end{array}$ & $\begin{array}{l}430 \\
(302)\end{array}$ & $\begin{array}{l}430 \\
(302)\end{array}$ & $\begin{array}{l}430 \\
(299)\end{array}$ & $\begin{array}{l}470 \\
(276)\end{array}$ & $\begin{array}{l}403 \\
(318)\end{array}$ & $\begin{array}{l}425 \\
(303)\end{array}$ & $\begin{array}{l}(312 \\
(307)\end{array}$ \\
\hline
\end{tabular}

${ }^{a} \lambda_{\max }$ is phosphorescence maximum of each FQ in $2 \mathrm{mM} \mathrm{PB}$ aqueous solutions and in $1 / 2(\mathrm{v} / \mathrm{v}) 2 \mathrm{mM} \mathrm{PB} / \mathrm{ethanol}$. ${ }^{\mathrm{b}} \mathrm{E}_{\mathrm{T}}$ was determined from the wavelength observed when the FQ phosphorescence determined at $77 \mathrm{~K}$ was ca. $10 \%$ of its maximum because it is not possible to identify the $0-0$ band from vibronic structure of the FQ phosphorescences. ${ }^{c}$ The values obtained in $2 \mathrm{mM} \mathrm{PB/ethanol} \mathrm{(1/2)} \mathrm{resulted} \mathrm{to} \mathrm{be} \mathrm{identical} \mathrm{at} \mathrm{different} \mathrm{drug} \mathrm{concentrations}\left(5 \times 10^{-6} \mathrm{M}\right.$ and $\left.5 \times 10^{-4} \mathrm{M}\right)$ as well as using $2 \mathrm{mM}$ PB/ethylene glycol (1/2) as solvent.

Figure 1A showed this effect in the phosphorescence emissions of CPX and PPA. The value of the emission maximum of each compound using two concentrations is shown in Table 1. By contrast, it was not detected any concentration effect when the quinolone derivatives were studied using ethanol or ethylene glycol aqueous mixtures (see results in Table 1). However, a blue-shift of the phosphorescence emission was observed for each compound by the presence of the organic polar mixtures (see the phosphorescence blue-shift observed for NFX and ENX in Figure 1B). Table 1 shows the $\mathrm{E}_{\mathrm{T}}$ values determined for all the quinolones in different media from their phosphorescence spectra. The energies were lower in buffered aqueous media than those obtained using organic polar media. 


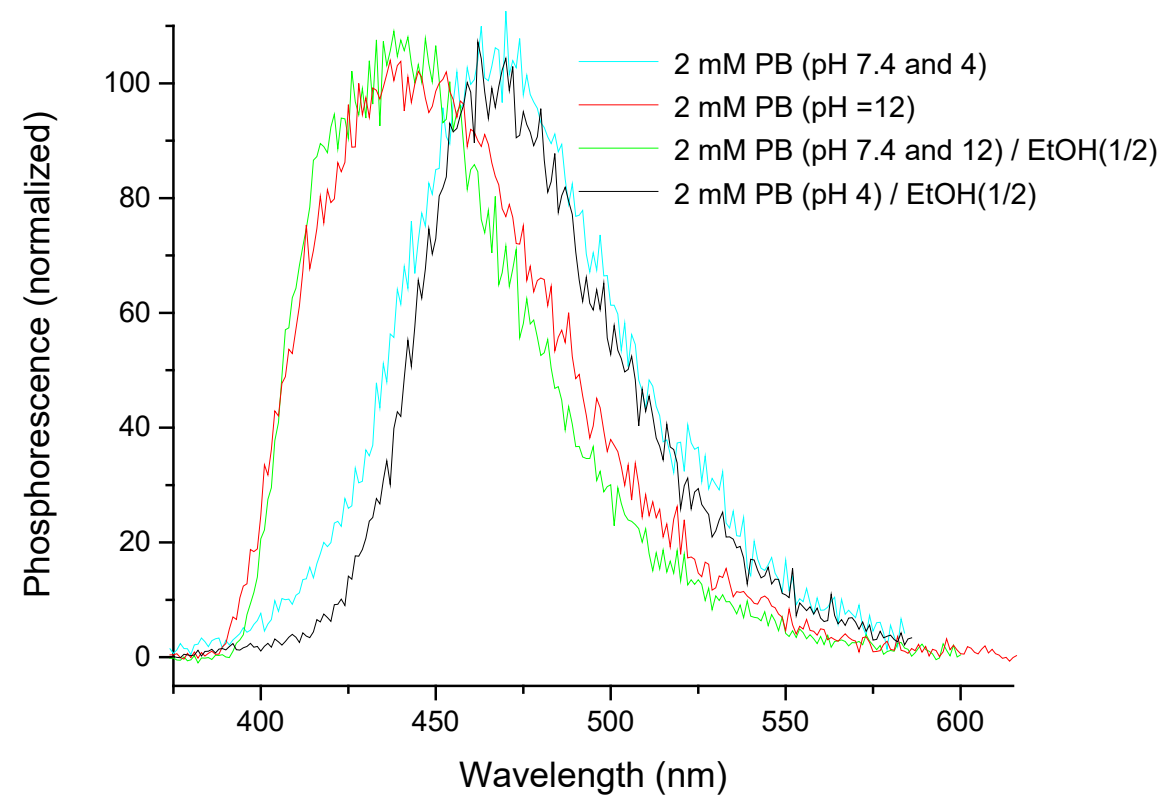

Figure 2. Normalized phosphorescence emissions of $\mathrm{CPX}\left(5 \times 10^{-6} \mathrm{M}\right)$ at $77 \mathrm{~K}$ in $2 \mathrm{mM}$ PB buffered water at pHs 7.4 (cyan) and 11 (red) and in organic aqueous mixtures of $2 \mathrm{mM}$ PB buffered water at neutral or acid pHs (7.4 and 4, respectively) with ethanol (1/2).

When low-temperature phosphorescence measurements of CFX were performed at different $\mathrm{pHs}$ in aqueous medium and in organic aqueous mixtures, similar blue-shift of the emission spectra was detected when de $\mathrm{pH}$ change from 4 to 12 (see Figure 2). The results in ethanol aqueous mixtures were identical than those obtained using the ethylene glycol mixtures (data not show). Interestingly, while the phosphorescence obtained at $\mathrm{pH}$ ca. 4 was the same than that observed at neutral $\mathrm{pH}$ in the aqueous medium, the emission spectrum obtained using the organic aqueous mixtures under neutral conditions ( $\mathrm{pH}$ 7.4) was similar to those registered when the solvents were under basic conditions.

\subsection{Effect of the amphoteric nature of quinolone derivatives in the excimer generation.}

Time-resolved nanosecond laser flash photolysis experiments at $355 \mathrm{~nm}$ excitation wavelength using $\mathrm{N}_{2} \mathrm{O}$ bubbled phosphate buffer (PB) solutions of two very similar quinolone derivatives were performed in order to detect the possible generation of their corresponding excimers. The chosen compounds for that proposal were PPA as an amphoteric compound and PRA as a simpler quinolone derivative without this property. The laser energies were attenuated to $<5 \mathrm{~mJ}$ pulse $\mathrm{e}^{-1}$ to minimize the two-photon ioniza- 
tion process, whose contribution could be important at higher pulse energies.17 Moreover, as commented above, $\mathrm{N}_{2} \mathrm{O}$ atmosphere was used to remove some solvated electron signal.[21]
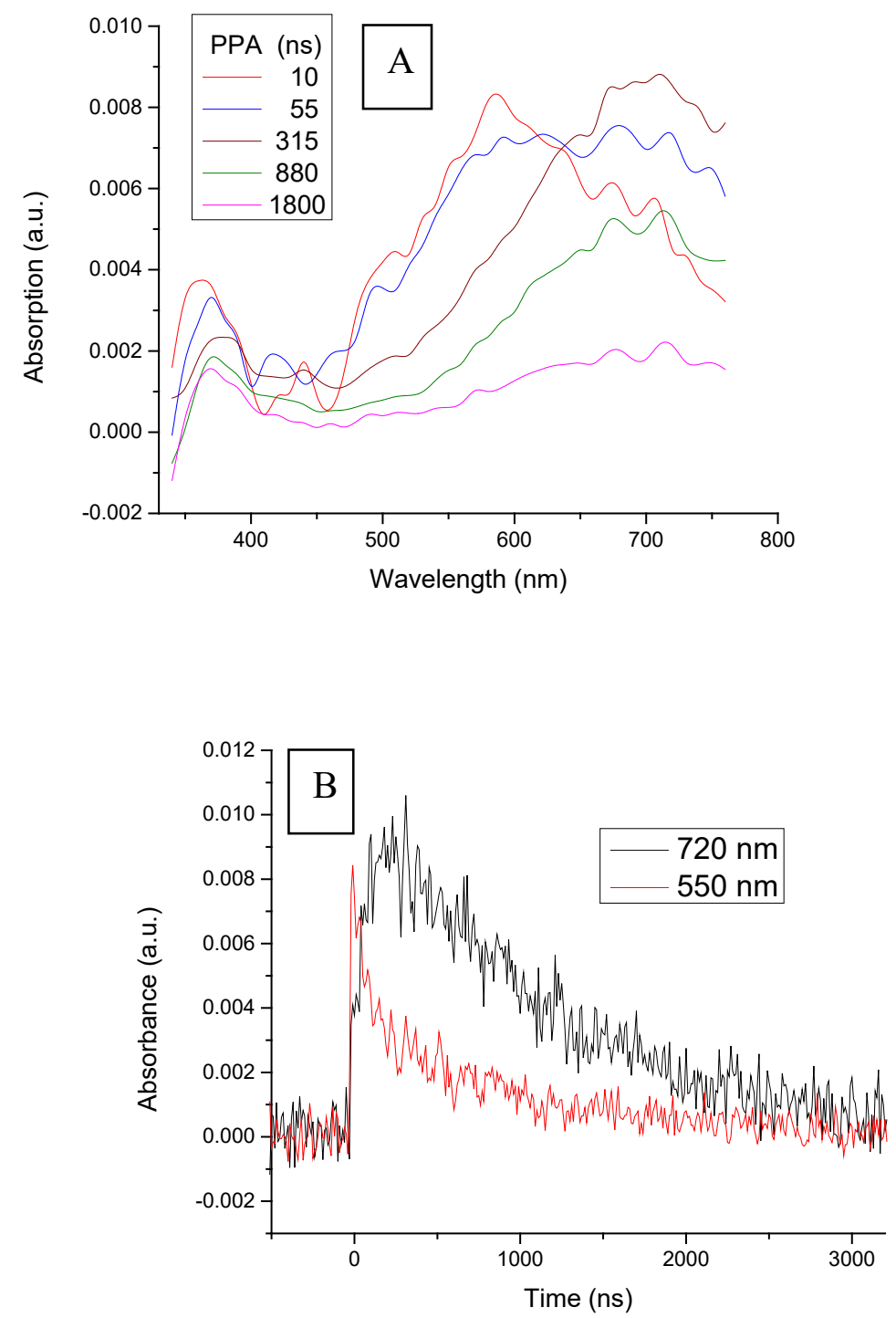

Figure 3. A) Transient absorption spectra of PPA $\left(10^{-4} \mathrm{M}\right)$ aqueous solutions under $\mathrm{N}_{2} \mathrm{O}$ atmosphere at different delay times after the laser pulse $\left(\lambda_{\text {exc }}=355 \mathrm{~nm}\right)$. B) Decay traces at 720 and $550 \mathrm{~nm}$ (lines black and red respectively).

Figure 3 shows the transient absorption spectra obtained for PPA at different times after de laser pulse. The maximum of the first transient species ( $\tau$ ca. $140 \mathrm{~ns}$ ) detected is very close to $580 \mathrm{~nm}$. A second transient with $\tau$ ca. $1420 \mathrm{~ns}$ arising from the first one can also be observed at $\lambda_{\max } 710 \mathrm{~nm}$. The first 
transient species was assigned as triplet excited state of PPA ( ${ }^{3}$ PPA) by using the energy transfer method (equation 1 in experimental section).
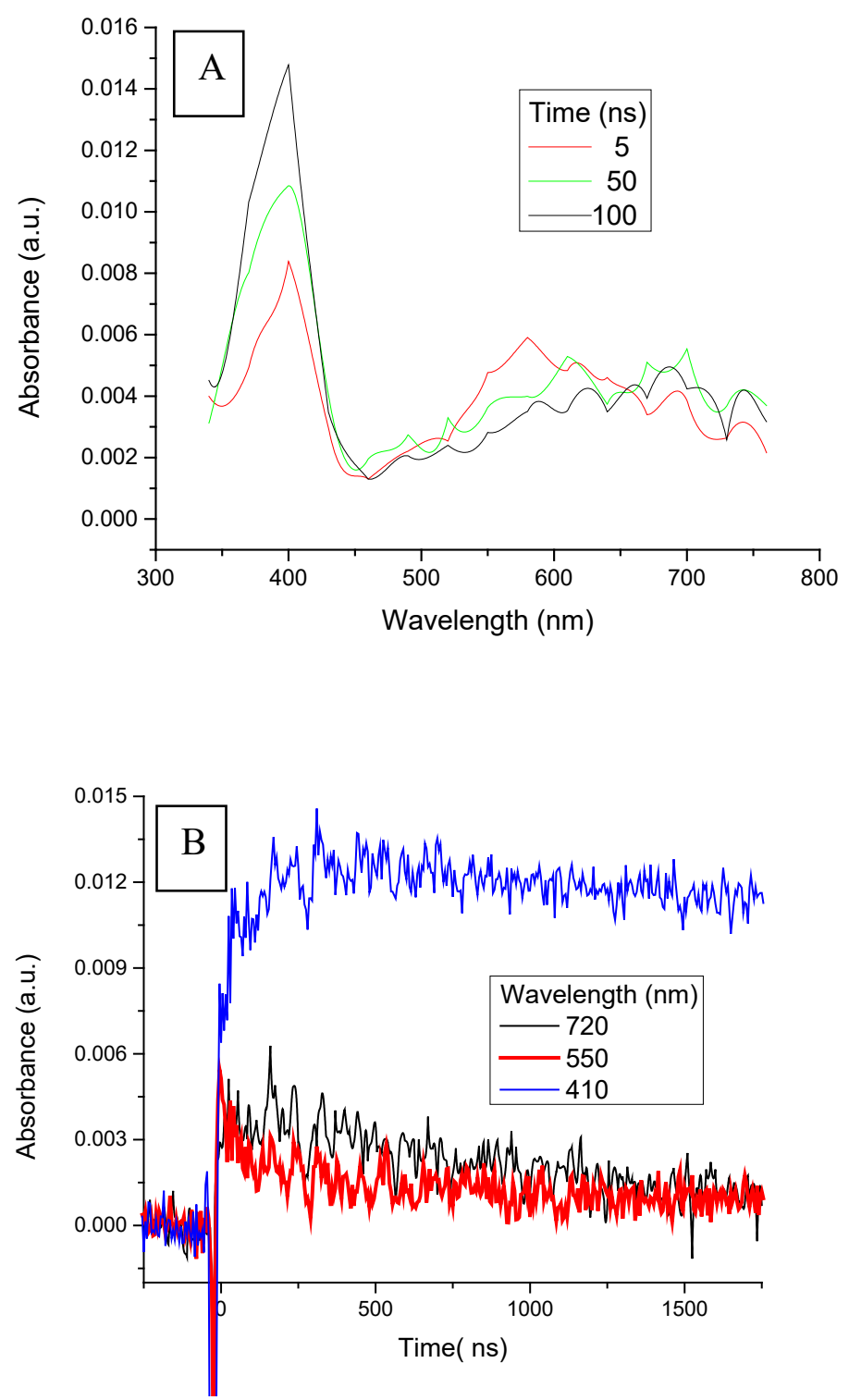

Figure 4. A) Transient absorption spectra of aqueous solutions of PPA $\left(10^{-4} \mathrm{M}\right)$ with BPC $\left(3.510^{-3} \mathrm{M}\right)$ under $\mathrm{N}_{2} \mathrm{O}$ atmosphere at different delay times after the laser pulse $\left(\lambda_{\text {exc }}=355 \mathrm{~nm}\right)$. B) Decay traces at 720, 550 and $410 \mathrm{~nm}$ (lines black, red and blue respectively).

Biphenyl carboxylic acid (BPC) was used as an energy acceptor compound. Thus, ${ }^{3} \mathrm{BPC}\left(\lambda_{\max } 410\right.$ $\mathrm{nm})[25]$ was generated from quenching of ${ }^{3} \mathrm{PPA}$ and an energy transfer rate constants of $210^{9} \mathrm{M}^{-1} \mathrm{~s}^{-1}$ 
was determined. In Figure 4A can be observed the transient absorption spectra detected at different times after the laser pulse. The decrease of the ${ }^{3} \mathrm{PPA}$ absorption simultaneously occurs with a growth of ${ }^{3} \mathrm{BPC}$ absorption. Thus, as shown in Figure 4B, the decay at $550 \mathrm{~nm}$, which corresponds to the ${ }^{3} \mathrm{PPA}$, is parallel with the growth of the signal displayed at $410 \mathrm{~nm}$, which is the $\lambda_{\max }$ of ${ }^{3} \mathrm{BPC}$. Moreover, generation of the transient species arising from ${ }^{3} \mathrm{PPA}$ (see trace at $720 \mathrm{~nm}$ ), its excimer, is reduced by adding BPC to the PPA aqueous solutions. This fact can be observed by checking the intensity ratios between 720/550 signals with and without BPC (Figure 4 and 3 respectively). Nevertheless, although lifetime of the excimer was not modified by the presence of BPC, this intermediate resulted to be efficiently quenched by molecular oxygen and a rate constant $\left(\mathrm{k}_{\mathrm{q}}\right)$ of ca. $210^{-9} \mathrm{M}^{-1} \mathrm{~s}^{-1}$ was determined.

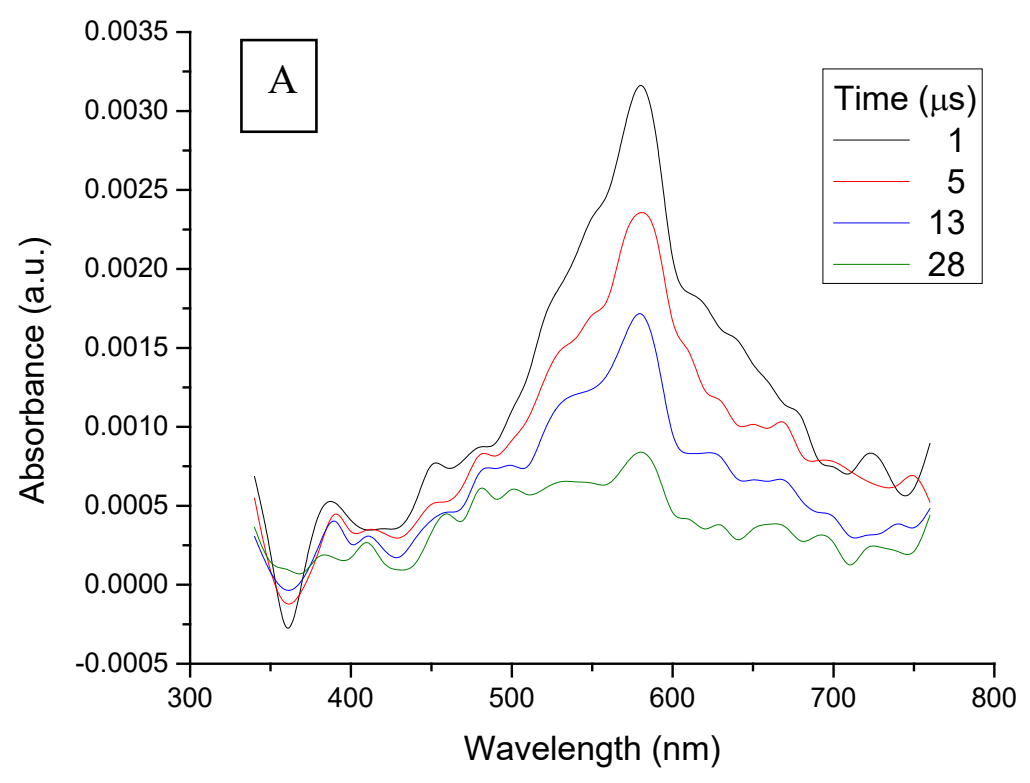




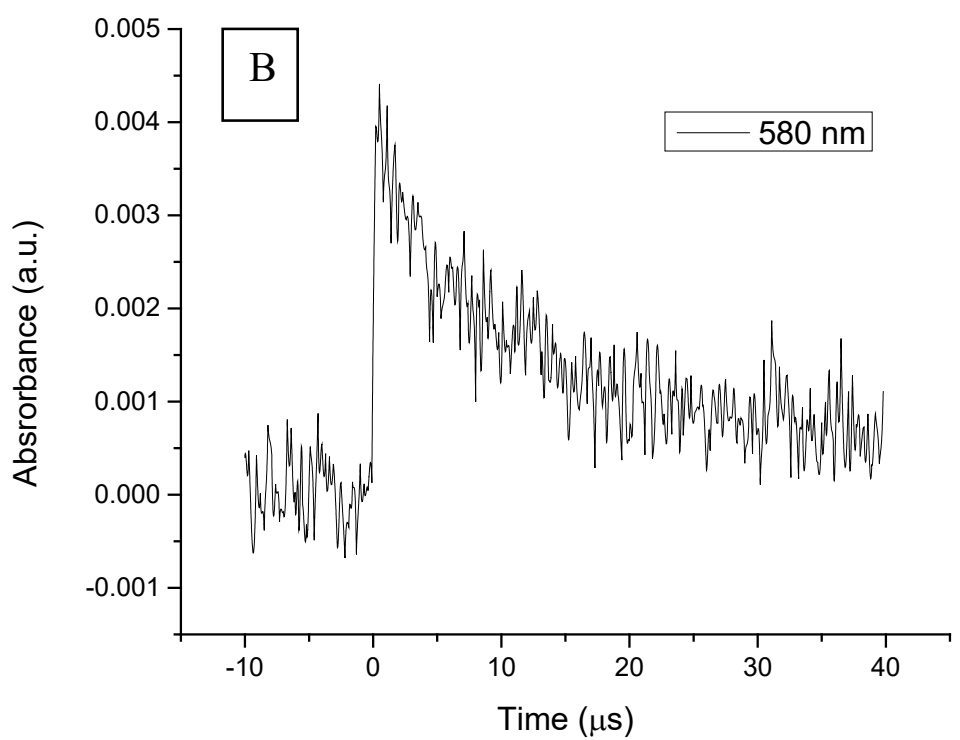

Figure 5. A) Transient absorption spectra of aqueous solutions of PRA $\left(10^{-4} \mathrm{M}\right)$ under $\mathrm{N}_{2} \mathrm{O}$ atmosphere at different delay times after the laser pulse $\left(\lambda_{\mathrm{exc}}=355 \mathrm{~nm}\right)$. B) Decay trace at $580 \mathrm{~nm}$ (black line).

When laser flash photolysis was performed for PRA in aqueous media under anaerobic conditions $\left(\mathrm{N}_{2} \mathrm{O}\right)$, only one transient absorption species $\left(\lambda_{\max } 580 \mathrm{~nm}\right.$ and $\tau$ ca. $\left.15 \mu \mathrm{s}\right)$ was detected (see Figure 5). The energy transfer study performed with PRA using BPC exhibited identical behavior with similar absorption spectra than that observed for PPA and the quenching rate constant was $110^{9} \mathrm{M}^{-1} \mathrm{~s}^{-1}$, which proves that the intermediate observed at $\lambda_{\max } 580 \mathrm{~nm}$ correspond to ${ }^{3} \mathrm{PRA}$. However, the generation of another intermediate was not detected in this study. 


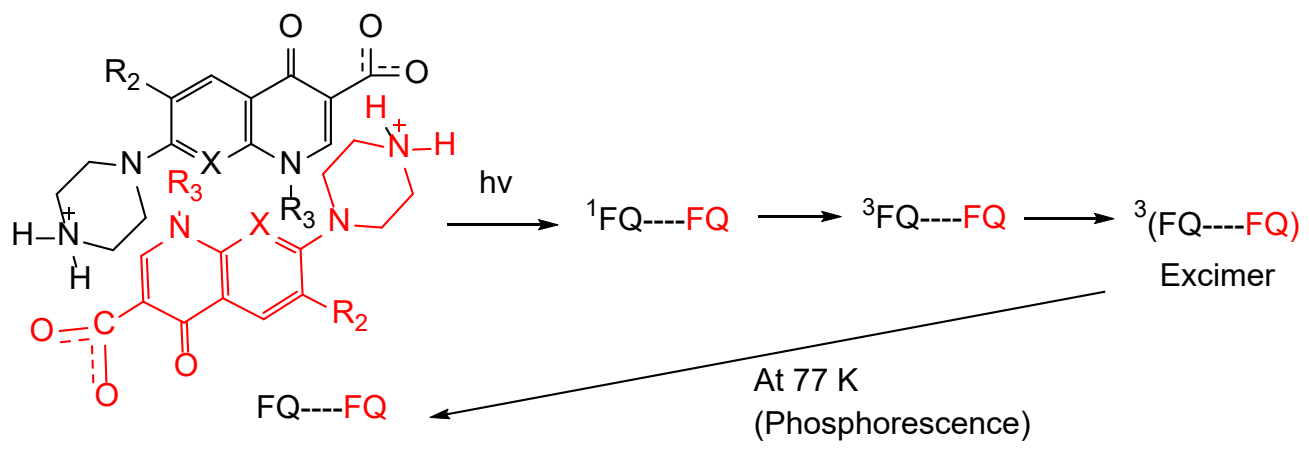

Scheme 1. Excimer generation pathway from FQ self-associations in buffered aqueous media.
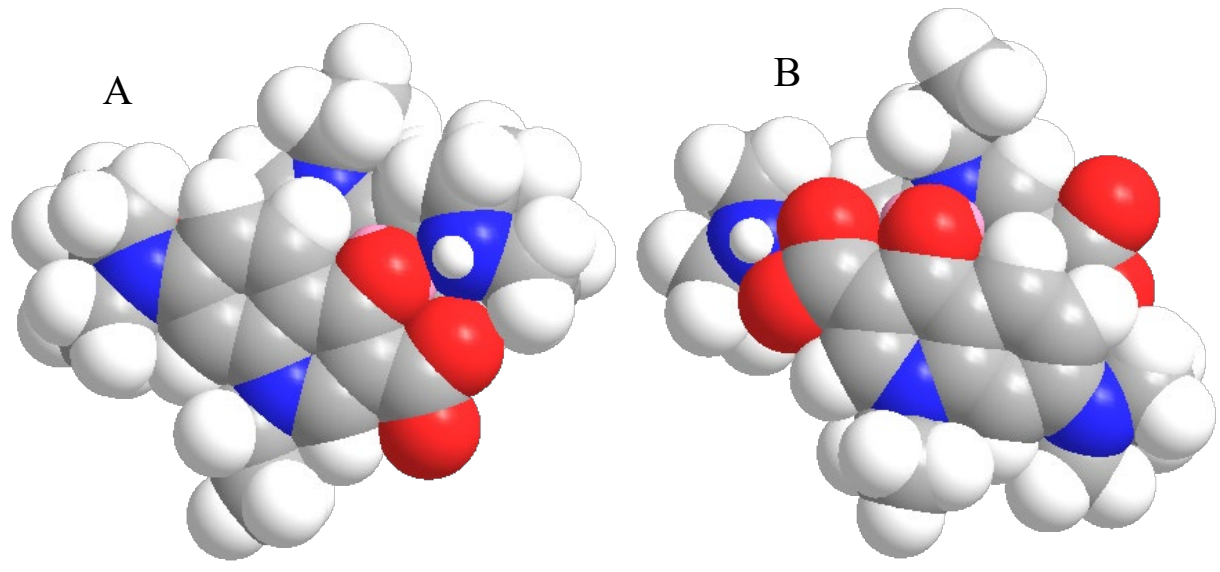

Figure 6. Most stable NFX-NFX association obtained using MM2 computation. A) Front view of NFX--NFX, B) Back view of NFX---NFX

\section{Discussion}

Low-temperature phosphorescence results of the present study have evidenced the crucial direct relation between the amphoteric nature of the quinolones and their self-associations. Thus, it was observed that the phosphorescence maxima of CPX, NFX, PFX, ENX, OFX and PPA obtained in buffered neutral aqueous media are redshift by increasing their concentrations while this effect was not observed for the anionic forms of NLA and PRA. Thereby, triplet excited state energy $\left(\mathrm{E}_{\mathrm{T}}\right)$ of the amphoteric quinolones is reduced increasing their concentrations in aqueous media (Table 1 shows the $\mathrm{E}_{\mathrm{T}}$ values of all the quinolones under different conditions). The self-association of the amphoteric compounds could explain 
the phosphorescence changes. The most stable association between two zwitterionic NFX molecules is shown in Figure 6, which is almost identical to the described for CPX using DFT calculation in the presence of water molecules.[3] Interestingly, while carbonyl at C4 and an one oxygen of the carboxylate at $\mathrm{C} 3$ of the front NFX are interacting with the hydrogens of the protonated nitrogen of the back molecule see Figure 6A), the interaction of the hydrogens of the nitrogen of the front NFX is taking place between the two oxygen of the carboxylate of the back molecule (see Figure 6B). Anyway, the close proximity of two molecules in the aqueous medium at $77 \mathrm{~K}$ could produce a high delocalization of the electrons of the phosphorescent chromophore when triplet excited state of one of the two molecules is generated by light (see in Scheme $\mathbf{1}$ is shown the pathway). This hypothesis was confirmed by performing the phosphorescence measurements using ethanol or ethylene glycol aqueous mixtures as solvents (see results in Table 1). Hence, the compounds did not show any phosphorescence change by increasing concentrations because, as expected, the presence of polar organic solvents must inhibit the self-associations of the amphoteric compounds. However, a blue-shift of the phosphorescence emission of each compound was detected when the measurements were performed in these organic polar solvents (see the phosphorescence blue-shift observed for NFX and ENX in Figure 2). The $\mathrm{E}_{\mathrm{T}}$ of FQs in these media were higher than those determined in buffered aqueous medium at neutral $\mathrm{pH}$. This result has been associated with a solvatochromic effect.[11] Nevertheless, the possibility of the involvement of a free carboxylated form of FQs in the organic mixtures (see form A in Scheme 2) and a cyclized form (see forms B and C) in buffered aqueous medium cannot be rolled out because blue-shifts of FQs fluorescence have also been observed when the $\mathrm{pH}$ changes from 3 to 7.[24-25] Thereby, low-temperature phosphorescence measurements using CFX in aqueous medium and in an organic polar mixture at $\mathrm{pHs}$ ca. 3 and 12 were performed to analyse this possibility. Phosphorescence blue-shift of CPX observed by changing the $\mathrm{pH}$ from 3 to 12 using the different solvents revealed the protic effect of the medium (see Figure 2). 


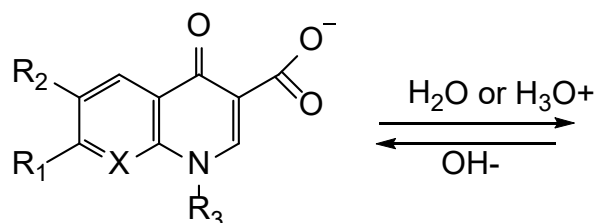

A

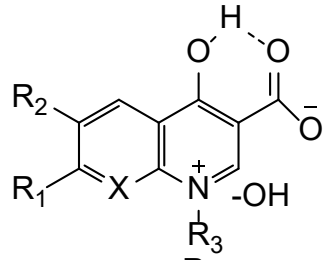

B

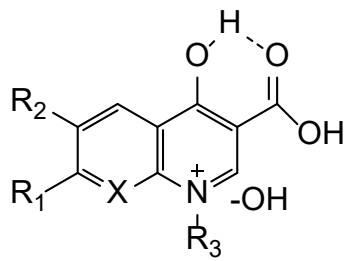

C

Basic media and organic polar buffered mixtures

Scheme 2. Quinolone derivative forms in different media at 77K.

Formation of a hydrogen bond between an oxygen of carboxylate at $\mathrm{C} 3$ and the carbonyl at $\mathrm{C} 4$ leading to a more extended electronic delocalization (cyclized form) could explain the FQs emissions obtained in the acidic media but also in buffered water (see forms C and B respectively in Scheme 2). By contrast, the open form of the quinolones would be the predominant structure in organic buffered polar mixtures as well as under the basic environments (form A). Accordingly, the cyclized form of FQs in aqueous medium would change to their open form when FQs are associated with DNA because their low temperature phosphorescence values resulted to be similar to those detected in organic polar buffered mixtures.[11] It is noteworthy that the $\mathrm{E}_{\mathrm{T}}$ determined for $\mathrm{FQs}$ in water, organic mixtures as well as into DNA[11] must be lower at room temperature than at $77 \mathrm{~K}$ because excitation of FQs produces structural rearrangements at room temperature but not in rigid low-temperature glasses where the ground and excited states of the quinolone derivatives have similar geometries.[11] Thereby, discrepancies described in the literature for the $\mathrm{E}_{\mathrm{T}}$ of $\mathrm{FQs}$ can be understood considering that changes of medium polarity or proticity as well as the temperature considerably modify their $\mathrm{E}_{\mathrm{T}}$ values.[11,14-21] Moreover, concentration changes of the amphoteric compounds also produces $\mathrm{E}_{\mathrm{T}}$ changes do to their self-associations in aqueous medium.

Concerning to these self-associations detected at low temperature, laser flash photolysis with two quinolones derivatives (PPA and PRA) were performed with the aim to prove that the excimer generation detected from FQ self-associations in aqueous media at room temperature can also be related to the amphoteric nature of these type of molecules and with the low-temperature phosphorescence findings. 
Thus, as all FQ self-associations described using laser flash photolysis technique have been done using FQs with the amphoteric nature, PPA and PRA were chosen for this study because they have the same quinolone-like chromophore but only PPA, with its 7-(1-piperazinyl) substituent, has an amphoteric nature. The triplet excited state nature of the first transient absorption species detected for PPA $\left(\lambda_{\max }\right.$ $580 \mathrm{~nm}$ ) was evidenced through an energy transfer reaction with BPC. The assignment of the transient species arising from ${ }^{3} \mathrm{PPA}$ as its triplet excimer $\left(\lambda_{\max }\right.$ ca. $\left.710 \mathrm{~nm}\right)$ was done by analogy to those described for NFX, PFX and related compounds.[8] Thus, it was observed that the PPA excimer shows a transient absorption spectrum with a maximum above $700 \mathrm{~nm}$ and it is efficiently quenched by molecular oxygen but not using BPC, which is in agreement with the photophysical properties described in the literature for other triplet excimers.[8, 26] Conversely, PRA triplet excited state did not generate any secondary transient species when the anionic form of PRA was studied under the same conditions and concentrations as those used for the amphoteric PPA. Interestingly, the lifetime of ${ }^{3} \mathrm{PRA}$ resulted to be ca. 100 times larger than that of PPA, which can be understood through a fast self-quenching of ${ }^{3} \mathrm{PPA}$ through its reaction with an associated PPA ground state (see in Scheme 1 the excimer generation pathway from FQ self-associations). Hence, the involvement of the amphoteric nature of the quinolone derivatives in the excimer generation has been clearly evidenced using these two compounds. Moreover, these data prove that FQ self-associations can be detected using the laser flash photolysis technique and performing low temperature phosphorescence measurements.

\section{Conclusion}

Low-temperature phosphorescence results showed that the amphoteric nature of the quinolones CPX, NFX, PFX, ENX, OFX and PPA is crucial to produce a redshift of their phosphorescence maxima by concentration increases in buffered aqueous medium. The presence of organic polar solvents in the buffered aqueous medium produced a blue-shift of the phosphorescence emission maximum of all quinolone derivatives. Formation of a cyclized form of FQ to lead to a more extended electronic delocalization 
would explain the low temperature emissions obtained in acidic solutions and in buffered water. By contrast, an open form of the quinolones would be the predominant structure in organic polar mixtures as well as under basic conditions. The correlation of the amphoteric nature of quinolone derivatives and their self-associations was also demonstrate performing a laser flash photolysis study with PPA and PRA where the generation of a triplet excimer was only detected for PPA. Moreover, the discrepancies described in the literature for the energy of the triplet excited sates of FQs can be understood considering that polarity and proticity of the media as well as the temperature can considerably modify their $\mathrm{E}_{\mathrm{T}}$ values. Thereby, it has been shown that low-temperature phosphorescence technique is an effective way to detect molecular self-associations and surrounding changes in quinolones, which opens the possibility to evaluate these effects in other drug families.

\section{Corresponding Author}

* Email: fbosca@,itq.upv.es

\section{Funding Sources}

Financial support from Spanish government (grant CTQ2014-54729-C2-2-P) and the Generalitat Valenciana (PROMETEO program, 2017-075).

\section{References}

[1] Ahmed, A., Daneshtalab, M., Nonclassical biological activities of quinolone derivatives. J.Pharm. Pharm. 15:52-72, 2012.

[2] Domagala, J. M., Hanna, L. D., Heifetz, C. L., Hutt, M. P., Mich, T. F., Sanchez, J. P., Solomon, M., New structure-activity relationships of the quinolone antibacterials using the target enzyme. The development and application of a DNA gyrase assay. J. Med. Chem. 29:394-404, 1986.

[3] Cramariuc, O., Rog, T., Javanainen, M.,Monticelli, L., Polishchuk, A. V., VattulainenaI., Biochim. Biophys. Acta 1818, 2563-2571, 2012.

[4] Sun, J., Sakai, S., Tauchi, Y., Deguchi, Y., Chen, J., Zhang, R., Morimoto, K., Determination of lipophilicity of two quinolone antibacterials, ciprofloxacin and grepafloxacin, in the protonation equilibrium. Eur. J. Pharm. Biopharm., 54, 51-58, 2002

[5] Sun, J., Sakai, S., Tauchi, Y., Deguchi, Y., Cheng, G., Chen, J., Morimoto, K., Protonation equilibrium and lipophilicity of olamufloxacin (HSR-903), a newly synthesized fluoroquinolone antibacterial. Eur. J. Pharm. Biopharm., 56, 223-229, 2003.

[6] Furet, Y.X., Deshusses, J., Pechère, J.C., Transport of pefloxacin across the bacterial cytoplasmic membrane in quinolone-susceptible staphylococcus aureus Antimicrob. Agents Chemother., 36, (1992) 2506-2511. 
[7] Maurer, N., Wong, K.F., Hope, M.J., Cullis, P.R., Anomalous solubility behavior of the antibiotic ciprofloxacin encapsulated in liposomes: a 1H-NMR study Biochim. Biophys. Acta, 1374, (1998) 9-20.

[8] Cuquerella, M. C., Andreu, I., Soldevila, S., Bosca, F. Triplet excimers of fluoroquinolones in aqueous media. J Phys Chem A 116, (2012) 5030-5038.

[9] Lhiaubet-Vallet, V., Sarabia, Z., Bosca, F., Miranda, M. A., Human Serum Albumin-Mediated Stereodifferentiation in the Triplet State Behavior of (S)- and (R)-Carprofen. J. Am. Chem. Soc. 126, (2004) 9538-9539.

[10] Bosca, F., Seeking to shed some light on the binding of fluoroquinolones to albumins. $J$ Phys Chem B 116, (2012) 3504-3511.

[11] Cuquerella, M. C., Lhiaubet-Vallet, V., Miranda, M.A., Bosca, F., Drug-DNA complexation as the key factor in photosensitized thymine dimerization. Phys. Chem. Chem. Phys 19, (2017) 4951-4955.

[12] Alfredson, T. V., Maki, A. H., Waring, M. J., Optically detected triplet-state magnetic resonance studies of the DNA complexes of the bisquinoline analogue of echinomycin. Biochemistry 30, (1991) 9665-9675.

[13] Alfredson, T. V., Maki, A. H., Phosphorescence and optically detected magnetic resonance studies of echinomycin-DNA complexes. Biochemistry 29, (1990) 9052-9064.

[14] Li, J., Li, J., Shuang, S., Dong, C., Study of the luminescence behavior of seven quinolones on a paper substrate, Anal. Chim. Acta 548, (2005) 134-142.

[15] Sun, C., Ping, H., Zhang, M., Li, H., Guan, F., Spectroscopic studies on the lanthanide sensitized luminescence and chemiluminescence properties of fluoroquinolone with different structure. Spectrochim. Acta A 82, (2011) 375-382.

[16] Rieutord, A., Vazquez, L., Soursac, M., Prognon, P., Blais, J., Bourget, P., Mahuzier, G., Fluoroquinolones as sensitizers of lanthanide fluorescence - application to the liquidchromatographic determination of ciprofloxacin using terbium. Anal. Chim. Acta 290, (1994) 215-225.

[17] Sortino, S., De Guidi, G.; Giuffrida, S.; Monti, S.; Velardita, A., pH effects on the spectroscopic and photochemical behavior of Enoxacin: A steady-state and time-resolved study. Photochem. Photobiol. 67, (1998) 167-173.

[18] Martinez, L.; Bilski, P.; Chignell, C. F., Effect of magnesium and calcium complexation on the photochemical properties of norfloxacin. Photochem. Photobiol. 64, (1996) 911-917.

[19] Bilski, P., Martinez, L. J., Koker, E. B., Chignell, C. F., Photosensitization by norfloxacin is a function of pH. Photochem. Photobiol. 64,(1996) 496-500.

[20] Bosca, F., Lhiaubet-Vallet, V., Cuquerella, M. C., Castell, J. V., Miranda, M. A., The Triplet Energy of Thymine in DNA. J. Am. Chem. Soc. 128, (2006) 6318-6319.

[21] Lhiaubet-Vallet, V., Cuquerella, M. C., Castell, J. V., Bosca, F., Miranda, M. A., Triplet Excited Fluoroquinolones as Mediators for Thymine Cyclobutane Dimer Formation in DNA. J. Phys. Chem. B 111,(2007) 7409-7414.

[22] Cuquerella, M. C., Lhiaubet-Vallet, V., Bosca, F., Miranda, M. A., Photosensitised pyrimidine dimerisation in DNA. Chem. Sci. 2, (2011) 1219-1232.

[23] Lhiaubet-Vallet, V., Bosca, F., Miranda, M. A., Photosensitized DNA damage: the case of fluoroquinolones. Photochem. Photobiol., 85 (2009), 861-868.

[24] Albini, A., Monti, S., Photophysics and photochemistry of fluoroquinolones. Chem. Soc. Rev. 32, (2003) 238-250.

[25] Cuquerella, M. C., Miranda, M. A., Bosca, F., Role of excited state intramolecular charge transfer in the photophysical properties of norfloxacin and its derivatives. J. Phys. Chem. A 110, (2006) 2607-2612.

[26] Lorenzo, F., Navaratnam, S., Allen, N. S. Formation of secondary triplet species after excitation of fluoroquinolonesin the presence of relatively strong bases. J. Am. Chem. Soc. 130, (2008) 12238-12239. 